This item was submitted to Loughborough's Research Repository by the author.

Items in Figshare are protected by copyright, with all rights reserved, unless otherwise indicated.

\title{
Multi-criteria decision making under uncertainty in building performance assessment
}

PLEASE CITE THE PUBLISHED VERSION

http://dx.doi.org/10.1016/j.buildenv.2013.07.019

PUBLISHER

(c) Elsevier

VERSION

AM (Accepted Manuscript)

LICENCE

CC BY-NC-ND 4.0

\section{REPOSITORY RECORD}

Hopfe, Christina J., Godfried L.M. Augenbroe, and Jan L.M. Hensen. 2019. "Multi-criteria Decision Making Under Uncertainty in Building Performance Assessment”. figshare. https://hdl.handle.net/2134/14706. 
This item was submitted to Loughborough's Institutional Repository (https://dspace.lboro.ac.uk/) by the author and is made available under the following Creative Commons Licence conditions.

\section{creative
commons}

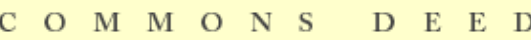

Attribution-NonCommercial-NoDerivs 2.5

You are free:

- to copy, distribute, display, and perform the work

Under the following conditions:

Attribution. You must attribute the work in the manner specified b the author or licensor.

Noncommercial. You may not use this work for commercial purposes.

No Derivative Works. You may not alter, transform, or build upon this work.

- For any reuse or distribution, you must make clear to others the license terms of this work.

- Any of these conditions can be waived if you get permission from the copyright holder.

Your fair use and other rights are in no way affected by the above.

This is a human-readable summary of the Leqal Code (the full license).

\section{Disclaimer 만}

For the full text of this licence, please go to: http://creativecommons.org/licenses/by-nc-nd/2.5/ 


\title{
MULTI-CRITERIA DECISION MAKING UNDER UNCERTAINTY IN BUILDING PERFORMANCE ASSESSMENT
}

\author{
Christina J. Hopfe ${ }^{a}$, G.A. Augenbroe ${ }^{b}$ and Jan L.M. Hensen ${ }^{c}$

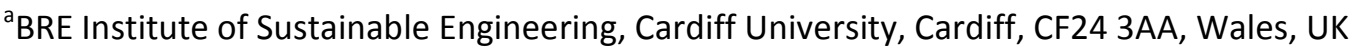 \\ ${ }^{\mathrm{b}}$ College of Architecture, Georgia Institute of Technology, Atlanta 30332-0155, USA \\ 'Building Physics and Systems, Eindhoven University of Technology, P.O. Box 513, NL-5600 MB, \\ Eindhoven, The Netherlands
}

\author{
Corresponding author: \\ Dr. Christina J. Hopfe \\ BRE Institute of Sustainable Engineering \\ Cardiff University \\ Queen's Buildings, The Parade \\ Cardiff CF24 3AA Wales, UK. \\ Tel: +44 2920870368 \\ Email: HopfeC@cardiff.ac.uk
}




\begin{abstract}
Building performance assessment is complex, as it has to respond to multiple criteria. Objectives originating from the demands that are put on energy consumption, acoustical performance, thermal occupant comfort, indoor air quality and many other issues must all be reconciled. An assessment requires the use of predictive models that involve numerous design and physical parameters as their inputs. Since these input parameters, as well as the models that operate on them, are not precisely known, it is imprudent to assume deterministic values for them. A more realistic approach is to introduce ranges of uncertainty in the parameters themselves, or in their derivation, from underlying approximations. In so doing, it is recognized that the outcome of a performance assessment is influenced by many sources of uncertainty. As a consequence of this approach the design process is informed by assessment outcomes that produce probability distributions of a target measure instead of its deterministic value. In practice this may lead to a "well informed" analysis but not necessarily to a straightforward, cost effective and efficient design process.

This paper discusses how design decision making can be based on uncertainty assessments. A case study is described focusing on a discrete decision that involves a choice between two HVAC system designs. Analytical hierarchy process (AHP) including uncertainty information is used to arrive at a rational decision. In this approach, key performance indicators such as energy efficiency, thermal comfort and others are ranked according to their importance and preferences. This process enables a clear group consensus based choice of one of the two options. The research presents a viable means of collaboratively ranking complex design options based on stakeholder's preferences and considering the uncertainty involved in the designs. In so doing it provides important feedback to the design team.
\end{abstract}

Keywords: Building performance simulation, multi-criteria decision making, uncertainty analysis, detailed design

\title{
1. Introduction
}

From evidence gathered from previous research [1], interviews with leading building and system designers, and design team observation it can be concluded that high performance buildings require an integrated team approach. Architects, engineers, building physicists, client, and occupants should be involved from the outset. Over-restricted, and/or non-synchronized design 
teams run the risk of limiting themselves too early in the design evolution [1;2;3;4]. However, even with well-coordinated partners, it can be difficult to find consensus on basic design concepts that lead to a design solution that all parties perceive as optimal. The main reason for this is the multitude of different perspectives, targets, criteria, vis-à-vis the preferences that are prioritised by each stakeholder. This situation begs for the adoption of rational decision making protocols by multi-stakeholder design teams as reported in $[5 ; 3 ; 6]$.

The implementation of decision making protocols in building performance is becoming more important and varies methods have been trialled in this regard [7;8;9]. For example, Attia et al, 2012 [7] describe the implementation of a simulation-based decision support tool in the conceptual design of zero-energy buildings in order to assess thermal comfort and energy performance options. Elsewhere, Bayesian decision theory has been used to support multicriteria decision-making [8]. This has been applied in the context of variable air volume (VAV) and constant air volume (CAV) systems in the isolation rooms in hospitals.

Most of the reported work does not deal with an important aspect of decision making: the role of uncertainty and the risk attitude of the stakeholders. We argue that uncertainty in performance predictions of competing options is rarely negligible and typically plays a major factor in the decision. At a certain level of granularity, design evolution can be viewed as a series of decisions under uncertainty. Every decision relies upon discrete design options that produce the most desirable outcomes, while accepting the associated risk that this option may also produce less favourable outcomes [10].

This situation suggests that a computational approach may be helpful in supporting a client/design team to reach an optimal decision. Wherein such an approach informs the design team about the predicted building performance (whilst also revealing the risk of underperformance) and forms the basis of a discussion about how to pinpoint the most favourable concept given the corresponding risk attitude of the stakeholders.

\section{The Decision making protocol}

Most decision making is prescriptive or normative. It is aimed at making the best decision without uncertainties arising. Decision makers should have the perfect insight and knowledge to take the most rational decision/ solution in the end. In the normative theory, a model is provided that allows a rational decision maker to keep his preference over certain attributes consistent in his task [11]. It enables the ranking of available options by decision maker's preference [11]. 
The deterministic problem can be expressed in a (comparison) matrix format that is shown in equation (1), where the criteria $C$ indicate the performance of the alternatives $A$.

$$
\begin{aligned}
& \begin{array}{llll}
w_{1} & w_{2} & . . w_{n}
\end{array} \\
& \begin{array}{llll}
C_{1} & C_{2} & . . C_{n}
\end{array} \\
& \begin{array}{cc}
x_{1} & A_{1} \\
x_{2} & A_{2} \\
\vdots & \vdots \\
x_{m} & A_{m}
\end{array}\left[\begin{array}{cccc}
a_{11} & a_{12} & . . & a_{1 n} \\
a_{21} & a_{22} & . . & a_{2 n} \\
a_{m 1} & a_{m 2} & . . & a_{m n}
\end{array}\right]
\end{aligned}
$$

A set of m alternatives $A_{1}, A_{2}, A_{3}, \ldots, A_{m}$ is given, as well as a set of $n$ decision criteria $C_{1}, C_{2}, C_{3}, \ldots, C_{n}$. Furthermore, it is assumed that the decision maker has determined the performance value $a_{i j}$ (for the $i^{\text {th }}$ alternative and the $j^{\text {th }}$ decision criterion) of each alternative; $w_{j}$ as the weight of the $j^{\text {th }}$ criterion and ${ }^{X_{i}}$ as the ranking value of the $i^{\text {th }}$ alternative of each alternative.

The weighting $w_{j}$ defines the importance of the criteria/ alternatives that takes into consideration each decision makers preference and risk attitude when evaluating design options to different performance aspects.

The Analytical Hierarchy Process (AHP) protocol (developed by Saaty in the 1970s) is one of the most widely applied and well-known techniques of Multi-criteria decision making (MCDM). AHP lets stakeholders rank the criteria by their importance in relation to the decision problem and in relation to each alternative through a pair-wise comparison [12].

It is based on the assumption that decision problems can be hierarchically structured with a one-directional relation between the decision levels. To the author's knowledge, there has been no research to date appraising methodological treatments of how and when design can be reduced to hierarchical decision making.

Whilst it can be stated that there is a design process involved in the making of every building and that process typically follows a common sequence (i.e. conceptual to detailed design stages) there is no agreed design assessment template that leads stepwise through this 
process. The reality shows that there is no conventional procedure; and that it may even be impossible to apply one [13;1]. The authors however take up the position that, since the design process commonly aims to satisfy a given set of targets, it is possible to translate this process into a decision path. Consequently this paper explores a hierarchical approach that is valid where the decomposition of identifiable targets are possible.

An important issue is the reciprocal consistency with respect the performance values in the comparison matrix, that involves the following hierarchical relationship: if $a_{i j}>1$ then $a_{j i}<1$ . Thus if $i$-th criterion dominates the $j$-th criterion, the $j$-th criterion cannot dominate the $i$-th criterion.

All of the comparisons among the different criteria, i.e., performance aspects are elements of the pairwise comparison matrix (as shown in Eq 1). The resulting weighting factors of the comparison matrix are all positive as they correspond to the relative ranking of the criteria. These weighting factors sum to unity as they are normalized. Thus, the relative ranking follows a linear order.

The decision making process involves ranking the alternatives and calculating a weighting factor. It is very important to note that when a new design option is added to a decision problem, the ranking of previous alternatives must not change, in order for rank reversal not to occur. This is one limitation of the AHP process, this is not discussed further however as it is assumed that all design options and alternatives have been agreed upon at the beginning of the decision making process by the different stakeholders.

The $A_{A H P s c o r e}^{*}$ of the best alternative is calculated by

$$
A_{\text {AHPscore }}^{*}=\max _{i} \sum_{j=1}^{n} a_{i j} w_{j}, \quad \text { for } i=1,2, \ldots, m \text { and } j=1,2, \ldots, n
$$

design alternatives $\left(A_{1}, A_{2}, \ldots, A_{m}\right)$

AHP is the most commonly applied technique in decision making in BPS. Chiang et al. [6] published a study on the comprehensive indicator of indoor environment assessment for occupants' health. Wong et al. [14] showed an application of the AHP in multi-criteria analysis of the selection of intelligent building systems. Kim et al. [15] developed a housing performance 
evaluation model for multi-family residential buildings considering criteria such as thermal comfort, indoor environmental quality, usability, and surroundings. In general it can be stated, that it is a very easy to implement approach, that is applicable for multiple stakeholders and multi-criteria decision problems. However, the classical AHP can be criticised because it lacks a firm theoretical basis, since uncertainties are not considered in the conventional MCDM approach.

The following sections introduce the case study, the design options and a decision making protocol in order to demonstrate a computational approach that informs the design team about predicted building performance. The treatment of the case follows mainstream rational decision theory [16] and hence assumes that the decision process is purely rational and that stakeholders pursue no other agenda then choosing the best performing design option, influenced only by the objective probabilistic predictions of the relevant performance measures, their (subjective) importance ranking and the risk attitude of each stakeholder. The decision problem thus falls into the category of MCDM under uncertainty [17]. It will be shown how this approach can be effectively applied to multi criteria and multi stakeholder decisions during design evolution.

\section{Case study}

"Het bouwhuis" is a building located in Zoetermeer, in the Netherlands between The Hague and Gouda, shown in Figure 1. It is the headquarters of Bouwend Nederland, the Dutch organisation of construction companies [18]. The building is an ideal case study because it combines flexibility and function. In addition the project's early stage confronted the design team with a choice between two distinct design options, both of which were developed in great detail.

The building process (from conceptual stage through to realisation of the building) took place from 2002 -2006. As mentioned earlier, two options were developed in great detail, i.e. both of them to production drawing stage. The first option represents a standard building services solution: using a conventional heating/cooling system. The second design option represents an innovative or potentially "risky" design, incorporating heating/cooling thermal storage in combination with a double façade. Both systems are described briefly below. 


\subsection{The Design options}

\section{Design option 1: Conventional heating/ cooling system}

Design option 1 uses conventional central heating and mechanical cooling; the building is conditioned by an air conditioning system with constant air volume (CAV) consisting of an air handling unit, supply and return fans, ducts and control units. Heating is provided by electrically driven radiators inside the room and an electric heater element in the air-handling unit (AHU). The system is regulated by air temperature control; during the office hours ( $8.00 \mathrm{am}-8.00 \mathrm{pm}, 5$ days per week) and is on night set back to standby mode the rest of the time. The AHU keeps the supply air temperature at $20^{\circ} \mathrm{C}$ when the incoming outside air temperature is below $16^{\circ} \mathrm{C}$ and can provide cooling, down to $27^{\circ} \mathrm{C}$ when the outside air temperature is above $40^{\circ} \mathrm{C}$. The ventilation system provides fresh air with a supply fan $\left(1000 \mathrm{~m}^{3} / \mathrm{h}\right)$ and exhausts the air by an exhaust fan $\left(1000 \mathrm{~m}^{3} / \mathrm{h}\right)$. The air change rate is 0.5 per hour. There is no night cooling.

\section{Design option 2: Heating/ cooling storage}

The second option presents an option for a building with a high percentage of glazing ( i.e. predominantly transparent facades): from the second floor up to the eleventh floor the building is on the gable end of each of three wings provided with a double façade (see Figure 2 and Figure 3). The outer skin of the double facade is built with one meter spacing from the internal façade of the building; thereby increasing the perimeter area of the building.

In winter the ventilation air is drawn in via the double skin façade, where it is naturally preheated, and then supplied as external air to the air handling unit. This method can be regarded as a heat-recovery system. In summer the double façade forms an extra barrier for solar radiation to enter the spaces as heat is removed from the façade air cavity through natural buoyancy driven ventilation to the outside. Another advantage is the increased noise attenuation performance of the double façade. The building is provided with a heat pump in combination with a heating-cooling storage system. Both systems (summer and winter) are demonstrated in Figure 4. The double glass façade is designed to have a positive influence on energy savings and to provide superior comfort. The material properties are identical for both options and therefore not explained in more detail. For more information please refer to [19].

\section{Simulation setup}

For the dynamic simulation of both alternatives, the following model characteristics and conditions are chosen to be identical in both options: 
- Internal heat gains: equipments $\left(20 \mathrm{~W} / \mathrm{m}^{2}\right)$; people $\left(10 \mathrm{~W} / \mathrm{m}^{2}\right)$ and lighting $\left(15 \mathrm{~W} / \mathrm{m}^{2}\right)$.

- Zoning: the assessment is conducted for the standard floor level comprising two zones for design option 1 and 5 zones for design option 2 (see Figure 2 and Figure 3).

- The assessment is based on the simulation of one room. All of the results presented relate to the smaller office room. The cavity between the glazing on the double skin is located at the south- facing surface of the building (see Figure 2 and Figure 3 ).

- Set Points: The indoor set point in the office is $27^{\circ} \mathrm{C}$ for cooling and $21^{\circ} \mathrm{C}$ for heating.

Both options are simulated in VA114. VA114 is a commercially available, industry strength, and extensively used BPS tool in The Netherlands.

\subsection{Design team and performance aspects}

The project design team consisted of the following members, amongst others: the architect [20], the building physics consultant and the building services/ systems engineer [21].

Three members of the design team were asked to make independent lists of the most important performance aspects of the building. Performance aspects such as initial costs, architectural layout, image/symbolism, energy consumption and thermal comfort were mentioned by all participants although with varying levels of significance and importance. Table 1 shows the (reduced set of) performance criteria that are the focus of the decision making process that is described in the next sections.

\subsection{The classical AHP}

In the classical AHP protocol the criteria have to be selected and ranked to each other by a pairwise comparison and assigning numbers from 1 as 'equally important' up to 9 for 'extremely more important' (see Table 2).

The matrix in Eq. 3 shows the result of the ranking based on a consensus process involving the three stakeholders. 
(3)

The result in Eq. 4 is the weighting factor based on normalizing all criteria after computing the Eigenvalue. As Saaty [22] has proven mathematically the Eigenvalue is a good solution for obtaining a set of priorities out of a pair-wise comparison matrix. Therefore, the matrix is multiplied with itself; the sum of the rows is built and normalized.

The normalized weights correspond to the relative dominance, and importance of each criterion, showing that 'Symbolism' ( $\mathrm{J}$ ) has the highest priority followed equally by 'Architectural form' (I), and the two Comfort criterion (over-and underheating hours $C$ and D).

$$
\left[\begin{array}{c}
B \\
C \\
D \\
E \\
F \\
G \\
H \\
I \\
J \\
K
\end{array}\right]=\left[\begin{array}{l}
0.061 \\
0.146 \\
0.146 \\
0.061 \\
0.018 \\
0.013 \\
0.061 \\
0.146 \\
0.287 \\
0.061
\end{array}\right]
$$

(4)

It can be noticed that the performance aspect A 'initial costs' is not included in Eq 4. Although it was stated that capital costs have an impact upon the final decision, the decision makers requested that costs be excluded at the outset of the decision protocol they only become 
relevant at the end of the decision making process. The purpose is thus to show graphically the overall performance compared to an independent cost factor in the final evaluation.

After calculating the weighting factor for the performance aspects $\mathrm{B}$ to $\mathrm{K}$ as shown in Eq. 3 and 4 , the two design options have to be compared to each other. For that reason they have to be assessed for every performance aspect separately. This is shown as an example for the 'architectural form' (I) in Eq. 5 and for 'symbolism' (J) in Eq.6. The perception for each option considering the criterion 'architectural form' (I) and 'symbolism' (J) is judged qualitatively by the stakeholders. As a result Design option 2 is considered to be (according to the stakeholders criteria weighting) "very strongly more important/ better" than Design option 1 . This relation is expressed with a ranking of ' 7 (cf. Table 2). The decision matrix is as follows.

$$
\begin{aligned}
& \begin{array}{ll}
\text { design } & \text { design } \\
\text { option } 1 & \text { option } 2
\end{array} \\
& {\left[\begin{array}{cc}
1 & 1 / 7 \\
7 & 1
\end{array}\right]^{2} ; \text { weighting factor } \text { Archticetural form }^{2}=\left[\begin{array}{l}
0.12 \\
0.88
\end{array}\right]}
\end{aligned}
$$

design option1 design option 2

This matrix results in a final ranking of the design option 1 for architectural form equals to 0.12 ; whilst design option 2 equals 0.88 .

This approach is identical for the performance aspect symbolism. Design option 2 is "strongly more important/ better" compared to design option 1 and is assigned with a ranking of ' 5 '.

$$
\begin{aligned}
& \text { design design } \\
& \text { option } 1 \text { option } 2 \\
& \begin{array}{l}
\text { design_option } 1 \\
\text { design option } 2
\end{array}\left[\begin{array}{cc}
1 & 1 / 5 \\
5 & 1
\end{array}\right]^{2} ; \text { weighting } \text { factor }_{\text {Symbolism }}=\left[\begin{array}{l}
0.17 \\
0.83
\end{array}\right]
\end{aligned}
$$

This matrix results in a final ranking of the design option 1 for symbolism equals 0.17 ; whilst design option 2 equals 0.83 .

Instead of weighting all performance aspects contingent upon the stakeholders individual preferences; the impact of subjective, and thus uncertain, information is reduced by the use of a simple numeric representation. This is done by including the outcome of a building performance 
simulation tool. Results such as energy consumption and thermal comfort are therefore calculated and inserted into the AHP protocol.

The approach is demonstrated in Table 3. The amount of weighted overheating hours for both options is inserted into the calculation and is further normalized. Hence, the weighting factor is based here on numerical performance data instead of users' preference.

The amount of weighted overheating hours for design option 1 calculated by the BPS tool is $17 \mathrm{~h}$ per year, for design option 2 it is $4 \mathrm{~h}$ per year. The italic part in Table 3 shows the calculation of the final rank for both options regarding the weighted overheating hours.

In the final step, the outcomes are summarized into one matrix. Table 4 shows a combination of subjective data based on expert preferences, experience, and personal judgement and on objective data from the simulation results provided by a BPS tool.

The values for performance aspects $\mathrm{B}, \mathrm{E}, \mathrm{G}, \mathrm{I}, \mathrm{J}$, and $\mathrm{K}$ are achieved based on the preferences and attitudes of decision makers according to Eq. 5 and 6. C, D and $\mathrm{H}$ are calculated by the BPS tool and normalized according to Table 3. The final outcome -based on the classical AHP protocol but with the help of Building Performance Simulation (BPS)- is for design option 10.23 and for design option 20.77 . This outcome shows that design option 2 is clearly more favourable than design option 1.

\subsection{The adapted AHP}

The traditional AHP protocol does not take into account that performance outcomes can be probabilistic variables. For the purpose of solving the application problem, we propose to extend the above method by adding uncertainty information. The goal is to include risk assessment in the conventional AHP protocol. Uncertainty analysis (UA) is applied to enable the designer to obtain an insight in to parameters chosen for each option. UA studies are conducted to show the variability in the output of a model that can be referred to different sources of variation in the input parameters.

In this study the emphasis has been on uncertainty in physical parameters, mostly identifiable as the standard input parameters in energy or thermal comfort simulation. Approximately 80 physical parameters have been considered in total. Assessments were made under fixed assumed use scenarios, which is common in uncertainty analyses. 
The most important parameter in design uncertainties is the room geometry. As the decision had to be made at a stage where the floor plan in either option was undefined, room geometry was entered into the uncertainty analysis. As an example, the outcome for annual heating is shown in Figure 5. The square shows the result of the first (deterministic) simulation - the result that is actually used in the conventional AHP protocol as shown above. The range gives an insight about the variability in outcome, i.e. how much impact uncertainties have on the simulation outcome after conducting 200 simulations.

It is important to add at this point that due to the early design choices made in this case study, there is an abundance of uncertainties some of which are quite large. This is what leads to the large uncertainty ranges in the outcomes of the results in Figure 5.

The conducted uncertainty analysis is studied quantitatively by assuming a normal distribution. The significance of this analysis in the informed use of BPS is therefore very high and could improve the decision process even though it might be argued that it is less credible to try to find an optimum when there are still so many open decisions with respect to design parameters . However, this is exactly the intention of this paper to find out whether this indeed leads to an outcome that has practical significance in some decision cases. The authors recommend that the inclusion of uncertainties is essential with respect to building simulation, performance, and design. The integration of uncertainties in BPS will provide an evidence based decision support process in design team meetings and dialogues with building partners.

Due to the consideration of uncertainties in the parameters, the simulation results for energy and thermal comfort cover a range as shown in Figure 5. The boundaries of this range can be titled as worst and best performance values due to the consideration of uncertainties. The worst and best performance also affects the weighting factor calculated in Table 1. How much either of them, the uncertainty and the weighting, impact the result is demonstrated in Table 5. The table shows the typical AHP result (from the classical method), and additionally the best and worst performance for both options in relation to the entire design team and each design team member separately. The results achieved are comparable to the conventional method but with the difference that hereby the upper and lower confidence bound of the results are taken to show the best and worst performance for energy and thermal comfort separately.

The percentage factor brings into relation the outcome of the simulation with the weighting calculated for energy and comfort. Due to the fact that the weighting differs for all three decision makers $(A, B$, and $C)$ the percentage is also affected. The first columns 'design option 1 and 2 
all' show the consensus of all three decision makers based on the weighting factor. The differences in performance listed in Table 5 can be also shown graphically. In Figure 6 the performance value is compared to a cost factor. For this purpose, the performance factor [\%] on the ordinate includes the calculated comfort from the simulation (weighted over-and underheating hours) and its confidence interval (due to the uncertainty range as seen in Figure 5 and 8) plus the subjective impact of all other performance aspects (as seen in Table 1 and calculated in Eq. 5 and 6).

Figure 6 shows the final output of the adapted AHP technique plus uncertainty protocol for comfort and energy factors. A performance value is then compared to a cost factor. The performance value includes all performance aspects considered except energy and costs. However, both, the energy consumption and the capital costs are excluded in this performance factor as they form part of the cost factor [\%] on the abscissa. The range in the performance is due to uncertainty and the weighting factor in the comfort prediction.

The cost factor is composed out of the capital costs for each building plus the running costs of energy consumption for each option over a chosen period of 5 years. Results are shown for all stakeholders separately in Figure 6. As it can be seen for all designers, design option 1 is the best performing alternative mainly because of its architectural form and its expected thermal comfort. However, design option 1 is also the more expensive solution due to its higher investment costs.

The range in the cost factor arises as a result of the uncertainty and the weighting in the energy consumption. The range in the performance is due to the uncertainty in the comfort criterion. By evaluating their impact on the final design- which is based on each decision maker individuallythese factors are normalized.

It is distinguishable (Figure 6) that there is a significant spread in the energy consumption whilst there is only a small spread in the cost factor. This is caused by the large difference in the initial costs (when the entire building costs are considered) which significantly outweigh the running costs (when compared over a 5 years term). Unless the building is only considered over a short lifespan, then the capital costs reflect only a minor percentage of the overall life costs and therefore play not a critical role in the overall assessment. 
The result is comparable to Figure 7. The difference is that instead of separating the outcomes based on the weighting of each decision maker, a consensus based on the weighting from Table 1 is built.

The risk involved with each option, given by the uncertainty range is shown in Figure 8.

The square in Figure 8 shows the results of the first simulation, the line with the barriers shows the results of the 200 simulations. The dashed line of the comfort criteria indicates whether the compliance with a certain requirement is exceeded. These thresholds are stated as expressions of minimally required performance for the weighted over- and underheating hours.

The output shows that for the better performing design (option 2) the amount of weighted underheating hours extends beyond the upper confidence bound of $150 \mathrm{~h}$ per year.

In order to diminish the uncertainty range, two possibilities will be pointed out: (1) Decreasing the risk of the scenario uncertainties, e.g., infiltration rate, and (2) adapting the design of the case study. The results will be shown for both approaches briefly:

\section{(i) Limiting the risk by controlling the scenario uncertainties}

Uncertainties in scenario conditions are very different from physical and design uncertainties in the sense that they can change during the building's life time [19]. Taking scenario uncertainties into account is relevant to design decision support, in particular when considering the design robustness and (future) adaptability of a building. These uncertainties originate from considering the wide range in the possible usage of a building typically referred to as usage scenarios. Scenarios encompass the influence of ventilation (the operation of window openings), climate change (for instance due to global warming), lighting control schemes, and other occupant related influences which result in unpredictable usage of the building.

One possibility is limiting the risk in the scenario uncertainties which means setting fixed limitations to boundary conditions. An example is the consideration of different user behaviour patterns related to operable windows. Another example is the change in the infiltration rate, which is considered to be varied between 0 and $0.2 \mathrm{ACH}$. This variation is assumed to be feasible as it encompasses a range which might be caused through bad workmanship or cracks in the façade.

As an example, the risk limitation will be carried out for the infiltration rate which has a corresponding linear dependency on the weighted under- and overheating hours (or vice versa). 
This is shown with the help of scatter plots. The creation of scatter plots is one of the simplest sensitivity analysis techniques. This approach consists of generating plots of the points

$\left(x_{i j}, y_{j}\right), i=1, \ldots, m$, for each independent variable $X i$ [23]. The purpose is to show the type of relationship or correlation that exists between two sets of data. The response variable is usually plotted on the vertical $Y$ axis, whilst the input variable is plotted on the horizontal $X$ axis.. Sometimes scatter plots completely reveal the relationship between model input and model predictions; this is often the case when there is only one or two monotonic inputs that dominate the outcome of the analysis [23], such as in the case of the relationship between thermal comfort and infiltration rate (see Figure 9).

The dashed line in Figure 9 indicates that limiting the infiltration rate to $0.8 \mathrm{ACH}$ will guarantee that the underheating performance threshold of $150 \mathrm{~h}$ per year is not exceeded. In this case, to eliminate the risk, the limitation of $0.8 \mathrm{ACH}$ must be fulfilled in order to avoid exceeding the confidence bound.

\section{(ii) Adapting the room size}

Design variations that occur during the planning process fall into the category of uncertainties in design parameters. They can be either caused by a lack of knowledge on the part of the designer or they may arise due to changes or irregularities in the planning phase of the building.

The weighted overheating hours are very sensitive to the geometry of the room. Nevertheless, there is no recognizable linear correlation to the weighted under- and overheating hours. For that reason, a new input file with different geometry data needs to be created and the uncertainty analysis needs to be conducted a second time. As a result a new simulation with a slightly decreased room size was initiated; the results are shown in Figure 10.

The uncertainty range for design option 2 is shown for the original performance and the performance after the changed room size for weighted over- and underheating hours. The number of exceeding weighted underheating hours is scaled down. From this it can be seen that improving the range of the design parameters in one direction can downgrade the uncertainty of another aspect, a consequence of which, in this case, is a slightly increased amount of overheating hours. However, it can be verified that by iteratively adding more realistic constraints on the parameter ranges of the options, the resulting conditional probabilities 
strengthen one option into the best option. Provided that, one option is optimal if it does not lead to unacceptable risk of underperformance as defined by the confidence bounds.

\section{Discussion}

Any methodological treatment of how and when design can be reduced to hierarchical decision making is still lacking. This paper positions itself that the design is satisfying a given set of targets, which is translated into a decision path. We used a hierarchical approach to explore its applicability to the group decision making under risk.

Thus, a conventional AHP protocol has been extended by the use of BPS and the integration of uncertainty analysis. Both, the conventional and the adapted AHP protocol fuse evaluations from multiple decision-makers with inconsistent viewpoints. The approaches average the multi objective evaluations to obtain a single consistent outcome (by having multiple decision makers). For weighting criteria or performance aspects, the traditional AHP uses a qualitative ranking. The adapted AHP methodology used here fuses subjective and objective information via numerical weighting. This approach allows balanced consideration of the following design criteria:

(i) Subjective or qualitative performance aspects such as the architectural layout.

(ii) Objective or quantitative performance aspects such as thermal comfort or energy consumption.

To handle performance aspects such as energy consumption, in the adapted AHP the output of BPS is used to include validated results into the decision process. Furthermore, the adapted AHP supports uncertain information. The conventional AHP protocol that handles only deterministic information is enhanced by uncertain building performance data through the use of uncertainty analysis. The sensitivity analysis in the adapted protocol is used to identify the most sensitive parameters that have the highest influence on the performance to eventually diminish the risk. The integration of uncertainty analysis supports risk identification as part of the decision process.

One drawback of the AHP protocol is with respect to rank reversal as mentioned in the beginning. There is however no plausible explanation for some of the rank reversals in AHP that can occur when something unrelated is changed. 


\section{Conclusion}

Current approaches in decision making for BPS do not integrate the combined use of simulation and uncertain information in the analysis of case study information. The adapted AHP protocol is used to expand current BPS capabilities to support the design team in making decisions. MCDM is herewith used to a lesser extent to indicate one solution as the best but to primarily show the impact on user preferences of a discrete set of options facing uncertainty. An advantage is that both subjective and objective evaluation measures are captured such as comfort, energy demand, and architectural layout.

The integration of a decision making protocol with the extension of uncertainty analysis in BPS can support the design process and provide additional information. It will help the design team in several ways, as follows.

(i) Support of the design team in the design process by providing a framework for communication.

(ii) Support in the decision process by providing a methodology to compare different design options.

(iii) Avoidance of pitfalls due to a lack of planning and a lack of a defined focus.

(iv) Possibility to minimise risk related to different concepts with the help of UA/SA.

(v) Understanding of how parameters are related to each other.

(vi) Comprehension of how variations in the model input affect the output.

(vii) Enhancement of the use of BPS by providing additional support, and therefore, leading to better guidance in the design process.

The developed approach is meant to enhance the information flow in an iterative design process as it shows the impact of uncertainty analysis embedded in a decision process. It is shown that the inclusion of uncertainties in BPS is essential with respect to simulation and performance. The integration of uncertainties in building performance can i provide evidence based decision support in design team meetings, thereby enhancing dialogues with project partners.

\section{Acknowledgement}

This research has been partly funded by the Vabi and Deerns.

We would like to thank Elphie Nelissen, Ed Rooijakkers, Hans Ruchti, Ivo Spierts, Paul Stoelinga, Jan Vambersky, and Jaap Wiedenhoff (alphabetically ordered) for their participation in our interviews. 


\section{REFERENCES}

[1] Hopfe, C., C. Struck, G. Harputlugil, J. Hensen, and P. de Wilde. 2005. Exploration of the use of building performance simulation for conceptual design. In Proceedings IBPSANVL Conference 20 October 2005 "Building Performance Simulation: a better support for the practice of today and tomorrow?". October 20.

[2] Hopfe, C. J., C. Struck, G. Ulukavak Harputlugil, and J. Hensen. 2006. Computational tools for building services design - professional's practice and wishes. 5. STP - Society of Environmental Engineering, Prague, May 17.

[3] Zavadskas, Edmundas Kazimieras, and Jurgita Antucheviciene. 2007. Multiple criteria evaluation of rural building's regeneration alternatives. Building and Environment 42, no. 1 (January): 436-451.

[4] Beccali, M., M. Cellura, and M. Mistretta. 2003. Decision-making in energy planning. Application of the Electre method at regional level for the diffusion of renewable energy technology. Renewable Energy 28, no. 13 (October): 2063-2087.

[5] Ugwu, O.O., and T.C. Haupt. 2007. Key performance indicators and assessment methods for infrastructure sustainability--a South African construction industry perspective. Building and Environment 42, no. 2 (February): 665-680.

[6] Chiang, Che-Ming, and Chi-Ming Lai. 2002. A study on the comprehensive indicator of indoor environment assessment for occupants' health in Taiwan. Building and Environment 37, no. 4 (April): 387-392.

[7] Shady Attia, Elisabeth Gratia, André De Herde, Jan L.M. Hensen, Simulation-based decision support tool for early stages of zero-energy building design, Energy and Buildings, Volume 49, June 2012, Pages 2-15, ISSN 0378-7788, 10.1016/j.enbuild.2012.01.028.

[8] Sean Hay Kim, Godfried Augenbroe, Decision support for choosing ventilation operation strategy in hospital isolation rooms: A multi-criterion assessment under uncertainty, Building and Environment, Volume 60, February 2013, Pages 305-318, ISSN 03601323, 10.1016/j.buildenv.2012.09.005.

[9] A.T. Booth, R. Choudhary, Decision making under uncertainty in the retrofit analysis of the UK housing stock: Implications for the Green Deal, Energy and Buildings, Available online 17 May 2013, ISSN 0378-7788, 10.1016/j.enbuild.2013.05.014.

[10] Maier, H. R., and J. C. Ascough II. Uncertainty in environmental decision-making: Issues, challenges and future directions, in Proceedings of the iEMSs Third Biennial Meeting: Summit on Environmental Modelling and Software [CD-ROM], edited by A. Voinov, A. Jakeman, and A. Rizzoli, Int. Environ. Modell. and Software Soc., Burlington, Vt.; 2006.(Available at http://www.iemss.org/iemss2006/sessions/all.html).

[11] Moon, Hyeun Jun, and Godfried Augenbroe. 2007. Application of the probabilistic simulation and Bayesian decision theory in the selection of mold remediation actions. In . Beijing, China. 
[12] Saaty, T.L. 1980. The Analytic Hierarchy Process, Planning, Piority Setting, Resource Allocation. McGraw-Hill,, New York.

[13] A Cemesova, CJ Hopfe, Y Rezgui, 2013. Client driven sensitivity analysis of the energy consumption of a Welsh office building using probabilistic climate projections. BS2013, Chambery, France

[14] Wong, Johnny K.W., and Heng Li. 2008. Application of the analytic hierarchy process (AHP) in multi-criteria analysis of the selection of intelligent building systems. Building and Environment 43, no. 1 (January): 108-125.

[15] Kim, Sun-Sook, In-Ho Yang, Myoung-Souk Yeo, and Kwang-Woo Kim. 2005. Development of a housing performance evaluation model for multi-family residential buildings in Korea. Building and Environment 40, no. 8 (August): 1103-1116.

[16] French S. (1986). Decision Theory: an Introduction to the Mathematics of Rationality. Chichester, Ellis Horwood.

[17] Novikova, N M, Pospelova, I I. Multicriteria Decision Making Under Uncertainty. Mathematical Programming. 2002; 92(3):537-554.

[18] Bouwend Nederland - Bouwhuis Zoetermeer. http://www.bouwendnederland.nl/perscentrum/beeldbank/bouwhuiszoetermeer/Pages/d efault.aspx (last accessed February 2009).

[19] Hopfe, C.J. \& Hensen, J.L.M., 2011. Uncertainty analysis in building performance simulation for design support. Energy and Buildings, 43(10), pp.2798-2805.

[20] klunder Architecten Rotterdam - home. http://www.klunderarchitecten.nl/ (last accessed December 2008).

[21] Nelissen ingenieursbureau bv. http://www.nelissenbv.nl/site/html/index.php (last accessed October 2008).

[22] Saaty, T., and K. Kearns. 1985. Analytical Planning: The Organization Of Systems. New York: Pergamon Press, Oxford.

[23] SimLab - Sensitivity Analysis. http://simlab.jrc.ec.europa.eu/ (last accessed January 2009).

\section{LIST OF FIGURES}

Figure 1 Illustration of "Het Bouwhuis"

Figure 2 Illustration of the footprint for design option 1 of "Het Bouwhuis" 
Figure 3 Illustration of the footprint for design option 2 of "Het Bouwhuis".

Figure 4 Illustration of the summer and winter case for design option 2 of "Het Bouwhuis" [Nelissen, 2008].

Figure 5 Range of the outcome for the annual heating obtained by 200 simulations considering physical, scenario and design uncertainties

Figure 6 Illustration of the results for the decision makers separately ( $A, B$, and C) comparing the cost factor to the performance.

Figure 7 Illustration of the results for a consensus of the decision makers comparing the cost factor to the performance

Figure 8 Illustration of the uncertainty range for annual heating, annual cooling and the weighted over- and underheating hours obtained by 200 simulations, showing the uncertainty range for (a) Annual Heating $\left[\mathrm{kWh} / \mathrm{m}^{2}\right]$, (b) Annual Cooling [kWh/m²], (c) Weighted underheating hours [h], and (d) Weighted overheating hours [h].

Figure 9 Scatter plot showing the relation between infiltration rate to weighted underheating (WOH-) and overheating hours ( $\mathrm{WOH}+$ ).

Figure 10 Illustration of the uncertainty range for weighted over- and underheating hours after the adaptation of the room size for design option 2.

\section{LIST OF TABLES}

Table 1 Listing of the performance aspects that are relevant in the decision making process Table 2 Illustration of the weighting in AHP (according to Saaty, 1980).

Table 3 Demonstration of the calculation of the importance weighting of the performance aspect C: weighted overheating hours.

Table 4 Ranking of both options for the performance aspects $\mathrm{B}$ to $\mathrm{K}$.

Table 5 Demonstration of the relation of the outcome energy and thermal comfort compared to the both options dependent on the user. 
Table 1 Listing of the performance aspects that are relevant in the decision making process.

\begin{tabular}{ll}
\hline A & initial costs \\
B & indoor resultant temperature \\
C & overheating hours (weighted) \\
D & under-heating hours (weighted) \\
E & individual control \\
F & floor area per person \\
G & space height \\
H & energy consumption \\
I & architectural form \\
J & symbolism (image /status) \\
K & changeability (flexibility)
\end{tabular}

Table 2 Illustration of the weighting in AHP (according to Saaty, 1980).

\begin{tabular}{lll}
$\begin{array}{l}\text { Intensity of } \\
\text { importance }\end{array}$ & Definition & Explanation \\
\hline \hline 1 & equally important & two elements have equal importance \\
\hline 3 & moderately more important & $\begin{array}{l}\text { experience or judgment slightly favors one } \\
\text { element }\end{array}$ \\
\hline 5 & strongly more important & $\begin{array}{l}\text { experience or judgment strongly favors one } \\
\text { element }\end{array}$ \\
\hline 7 & $\begin{array}{l}\text { very strongly more } \\
\text { important }\end{array}$ & dominance of one element proved in practice \\
\hline 9 & extremely more important & $\begin{array}{l}\text { the highest order dominance of one element } \\
\text { over another }\end{array}$ \\
\hline
\end{tabular}

\footnotetext{
${ }^{1}$ Please note that if an performance aspect 1 has a number assigned to when compared to another performance aspect 2 , then 2 has the reciprocal value when compared with 1 , i.e., $a_{i, j}=1 / a_{j, i}$ so, for example if $a_{i, j}=3$, then $a_{j, i}=1 / 3$
} 
Table 3 Demonstration of the calculation of the importance weighting of the performance aspect $C$ : weighted overheating hours.

\begin{tabular}{llll}
\multicolumn{5}{l}{ C: weighted overheating hours } \\
\hline \hline \multicolumn{5}{l}{$[\mathrm{h}]$} \\
\hline design option 1 & 17 & $1-(17 / 21)$ & 0.19 \\
design option 2 & 4 & $1-(4 / 21)$ & 0.81 \\
\hline \multicolumn{5}{l}{21} & &
\end{tabular}

Table 4 Ranking of both options for the performance aspects B to $\mathrm{K}$.

\begin{tabular}{llllllllllll} 
& B & C & D & E & F & G & H & I & J & K & Weighting factor \\
\hline \hline $\begin{array}{l}\text { design } \\
\text { option 1 } \\
\text { design } \\
\text { option 2 }\end{array}$ & 0.50 & 0.19 & 0.19 & 0.50 & 0.25 & 0.50 & 0.42 & 0.13 & 0.17 & 0.25 & $\mathbf{0 . 2 3}$ \\
\hline
\end{tabular}

Table 5 Demonstration of the relation of the outcome energy and thermal comfort compared to the both options dependent on the user.

\begin{tabular}{|c|c|c|c|c|c|c|}
\hline & & FS [\%] & $\begin{array}{l}\text { BP energy } \\
\text { [\%] }\end{array}$ & $\begin{array}{l}\text { WP energy } \\
\text { [\%] }\end{array}$ & $\begin{array}{l}\text { BP thermal } \\
\text { comfort [\%] }\end{array}$ & $\begin{array}{l}\text { WP thermal } \\
\text { comfort [\%] }\end{array}$ \\
\hline \multicolumn{7}{|l|}{ design } \\
\hline option 1 & all & 23 & 23 & 23 & 25 & 21 \\
\hline \multicolumn{7}{|l|}{ design } \\
\hline option 2 & all & 77 & 77 & 77 & 75 & 79 \\
\hline \multicolumn{7}{|l|}{ design } \\
\hline option 1 & A & 25 & 25 & 25 & 26 & 24 \\
\hline \multicolumn{7}{|l|}{ design } \\
\hline option 2 & A & 75 & 75 & 75 & 74 & 76 \\
\hline \multicolumn{7}{|l|}{ design } \\
\hline option 1 & B & 36 & 35 & 35 & 37 & 34 \\
\hline \multicolumn{7}{|l|}{ design } \\
\hline option 2 & B & 64 & 65 & 65 & 63 & 66 \\
\hline \multicolumn{7}{|l|}{ design } \\
\hline option 1 & C & 24 & 24 & 24 & 26 & 23 \\
\hline \multicolumn{7}{|l|}{ design } \\
\hline option 2 & C & 76 & 76 & 76 & 74 & 77 \\
\hline \multirow[t]{4}{*}{ Legend } & & FS & \multicolumn{4}{|c|}{ first simulation } \\
\hline & & $W P$ & \multicolumn{4}{|c|}{ worst performance } \\
\hline & & $B P$ & \multicolumn{4}{|c|}{ best performance } \\
\hline & & $A, B, C$ & \multicolumn{4}{|c|}{ Different users } \\
\hline
\end{tabular}




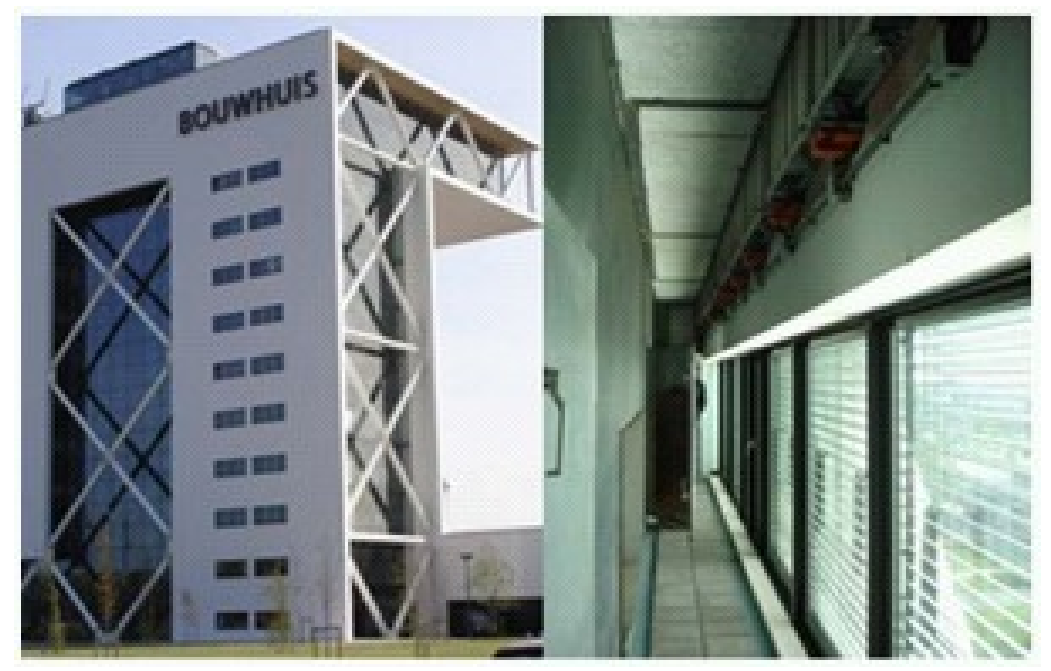

Figure 1

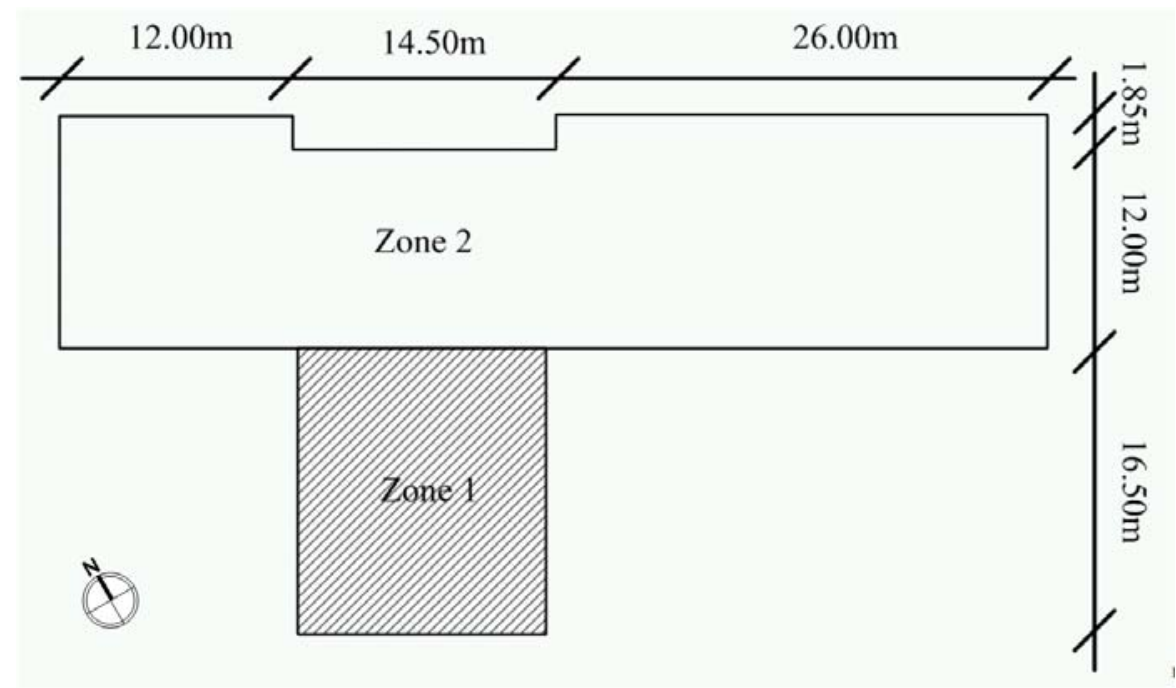

Figure 2 


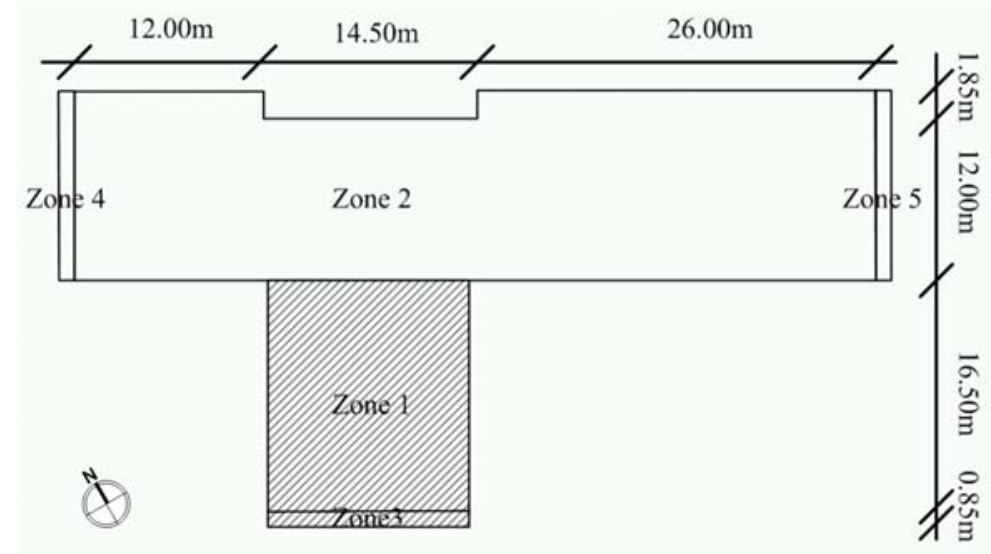

Figure 3

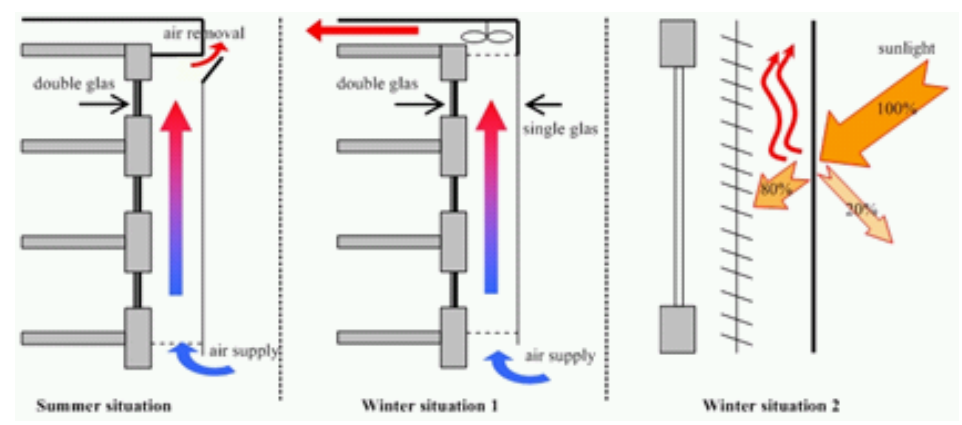

Figure 4

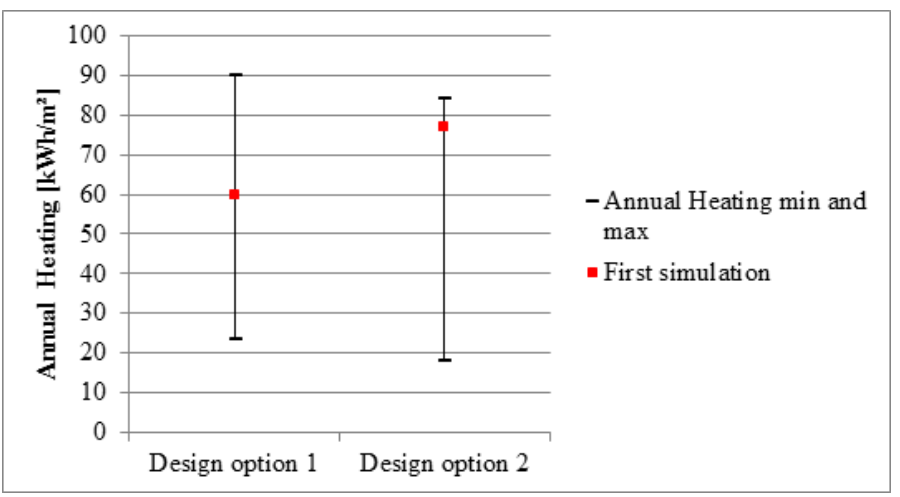




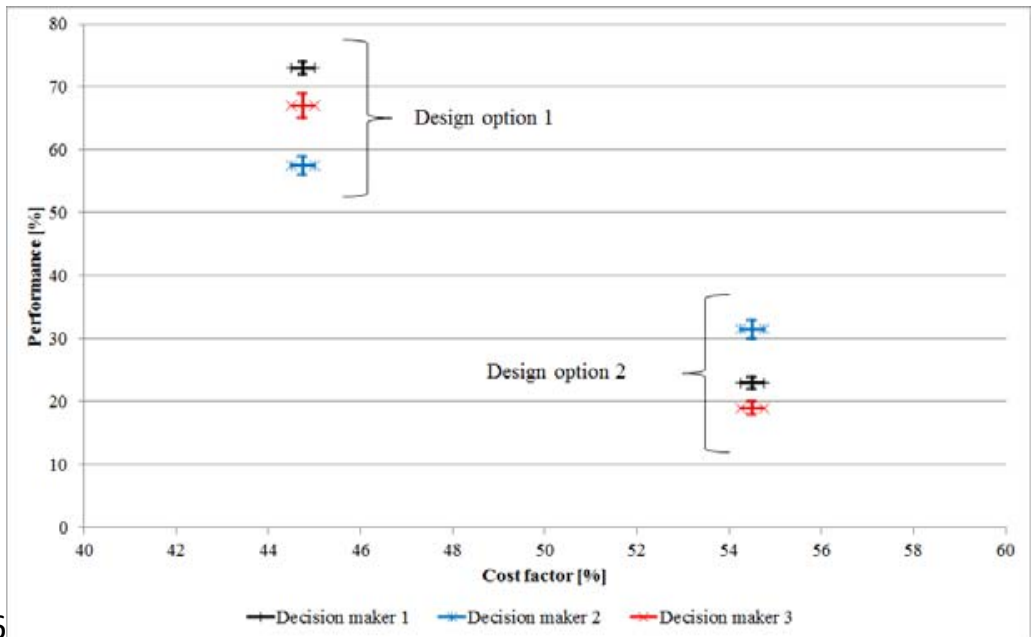

Figure 6

Figure 6

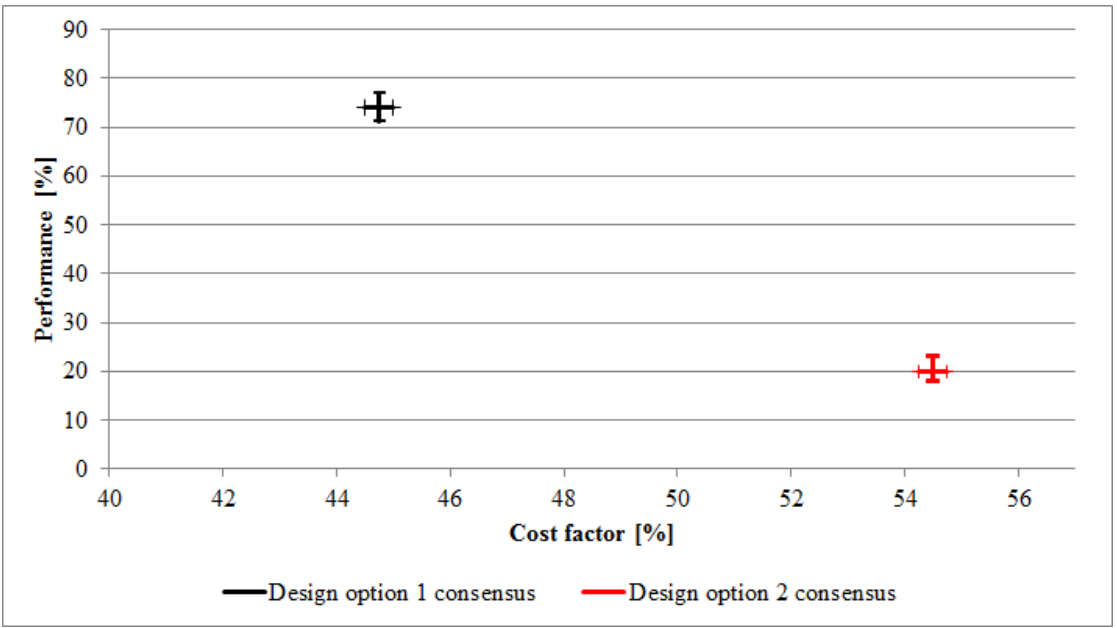

Figure 7 

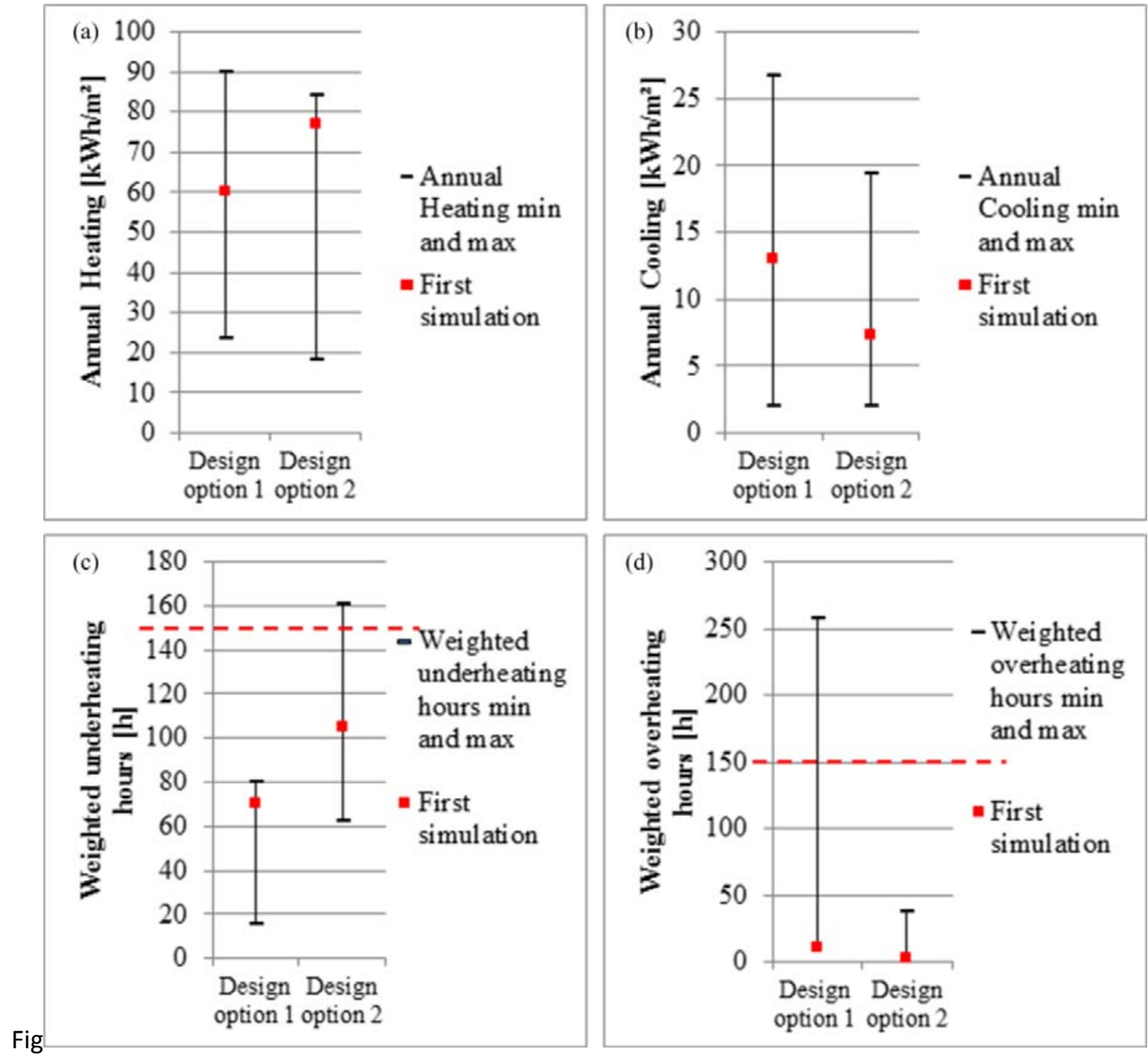

Figure 8 


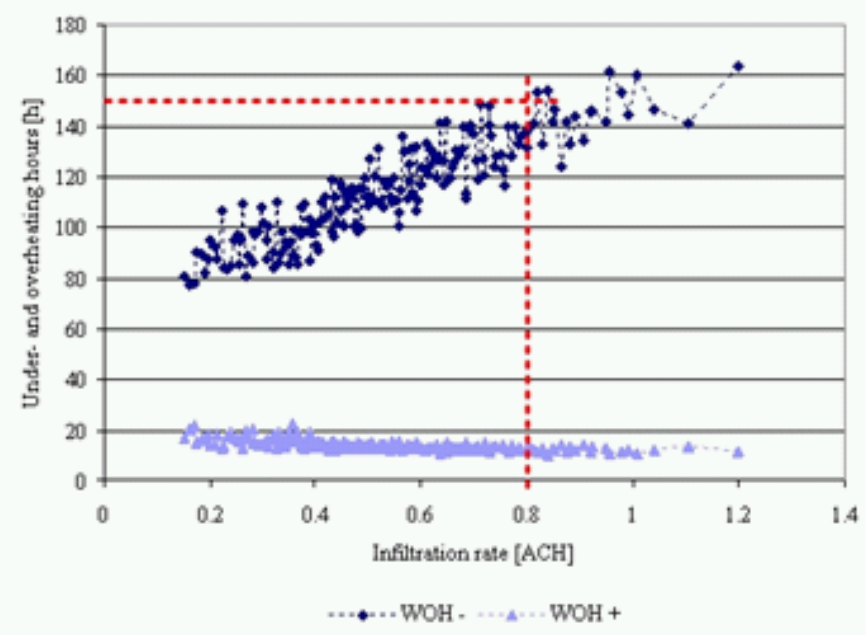

Figure 9 